\title{
Dynamics of the Plasma Column of a Capillary Discharge Soft X-Ray Laser
}

\author{
F. G. Tomasel, J. J. Rocca, and V. N. Shlyaptsev
}

\begin{abstract}
A sequence of time-resolved soft X-ray pinhole images shows the rapid plasma compression in a fast highpower capillary discharge. The compressed plasma column has a length-to-diameter ratio $>500$ and a good axial uniformity, characteristics that have allowed for the first demonstration of large soft X-ray amplification in a discharge-created plasma.
\end{abstract}

$\mathbf{U}$ NTIL recently and since their discovery a decade ago [1], [2], all soft $X$-ray lasers required of a laser created plasma. We recently realized the first demonstration of large soft X-ray amplification in a plasma column generated by an electrical discharge [3], [4]. Fast discharge excitation of an argon-filled capillary channel was used to compress the discharge power into small diameter plasma channels, simultaneously achieving the high-power density deposition and the good axial uniformity necessary to obtain amplification in the $J=0-1$ line of Ne-like Ar at $46.9 \mathrm{~nm}$. The most recent experiments have achieved a gain-length product of $g l \sim 14$ in this transition [5], the largest amplification obtained to date from a table-top soft X-ray amplifier.

The dynamics of the plasma column of the fast capillary discharge soft X-ray amplifier is illustrated in Fig. 1, which consists of a sequence of end-on soft X-ray pinhole camera images of the plasma. They were generated by imaging the capillary plasma into a multichannel plate (MCP) through a $45-\mu \mathrm{m}$ diameter pinhole placed on the capillary axis, at $36 \mathrm{~cm}$ from the end of the plasma column. The electrons emitted by the MCP were accelerated toward a phosphorous screen, and the resulting visible image was recorded with a charge-coupled device (CCD) array. The setup had a magnification of 3.1 and a spatial resolution that, depending on the wavelength of the radiation, was limited either geometrically by the size of the pinhole (to about $60 \mu \mathrm{m}$ ) or by diffraction. To produce each time-resolved image, a fast voltage pulse was applied to the MCP at selected time delays with respect to the initiation of the current pulse. The time resolution was approximately $5 \mathrm{~ns}$. To limit the detected radiation to wavelengths $\leq 30 \mathrm{~nm}$, a 100 $\mathrm{nm}$ thick carbon filter was used. The study was conducted in a 4-mm diameter $12-\mathrm{cm}$ long polyacetal capillary excited with a current pulse of $62 \mathrm{~ns}$ half period. The discharge conditions were near optimum for amplification: an $\mathrm{Ar}$ pressure of

Manuscript received August 10, 1995; revised October 13, 1995. This work was supported by NSF Grants ECS-9412916 and ECS-9401952, and in part by the U.S. National Research Council.

F. G. Tomasel and J. J. Rocca are with the Department of Electrical Engineering, Colorado State University, Fort Collins, CO 80523 USA.

V. N. Shlyaptsev is with the P. N. Levedev Physical Institute, Moscow, Russia.

Publisher Item Identifier S 0093-3813(96)02185-6.
700 mtorr and peak current of about $39 \mathrm{kA}$. The pinhole images clearly demonstrate that the plasma of the gas-filled fast capillary discharge rapidly contracts, heats up, and then expands, constituting a kind of wall influenced $Z$-pinch. Following the excitation of the pre-ionized plasma column with a fast voltage pulse, the current starts flowing near the capillary walls, in a region determined by the skin depth. During the first part of the current risetime, the distribution of the current density remains localized near the capillary wall. The first pinhole image [Fig. 1(A)], acquired $26 \mathrm{~ns}$ after the initiation of the fast current pulse, shows that the majority of the soft X-ray incoherent emission originates from a doughnutshaped region having an outer diameter of $3.5 \mathrm{~mm}$. After this initial phase, the electromagnetic forces of the rapidly rising current pulse create a shock wave and compress the plasma. The soft X-ray emitting region is rapidly compressed in about $10 \mathrm{~ns}$ to form a column $200-300 \mu \mathrm{m}$ diameter [Fig. 1(A)-(D)]. Hydrodynamic/atomic model calculations [6] show that during the compression and in advance of the collapse of the plasma sheath on the capillary axis, the current flow distribution switches from the periphery to the central region of the capillary, heating and ionizing the plasma, and increasing the soft X-ray emission near the axis. The emission reaches its maximum when the shock wave reaches the axis, causing the conversion of ion kinetic energy into thermal energy and a sharp increase in the electron density [Fig. 1(D)]. Lasing occurs during about $1 \mathrm{~ns}$ shortly before the time of maximum compression which occurs several nanoseconds after the peak of the current pulse. At the time of lasing, the electron density is about $0.3-1 \times 10^{19} \mathrm{~cm}^{-3}$ and the electron temperature reaches $60-90 \mathrm{eV}$. The pinhole images [Fig. 1(E)-(G)] show that subsequently, the plasma column expands and cools. A second less significant compression occurs near the end of the first cycle of the current pulse [Fig. 1(H)]. This time, a central column with a minimum diameter of about $1 \mathrm{~mm}$ and cooler temperatures, which are not of interest for the excitation of collisionally pumped soft X-ray lasers, is observed to develop on top of a broad plasma background. The pinhole images acquired with $(\lambda \leq 30 \mathrm{~nm})$ or without $(\lambda \leq 130 \mathrm{~nm})$ the carbon filter are similar except near the time of plasma collapse, when the full width at half maximum (FWHM) diameter of the radiating region are $200 \mu \mathrm{m}$ and $300 \mu \mathrm{m}$, respectively.

Amplification experiments conducted in plasma columns up to $20 \mathrm{~cm}$ in length [3], [5], the experiments discussed herein, and other studies show that the fast capillary discharge plasmas columns have high compression stability and symmetry among 

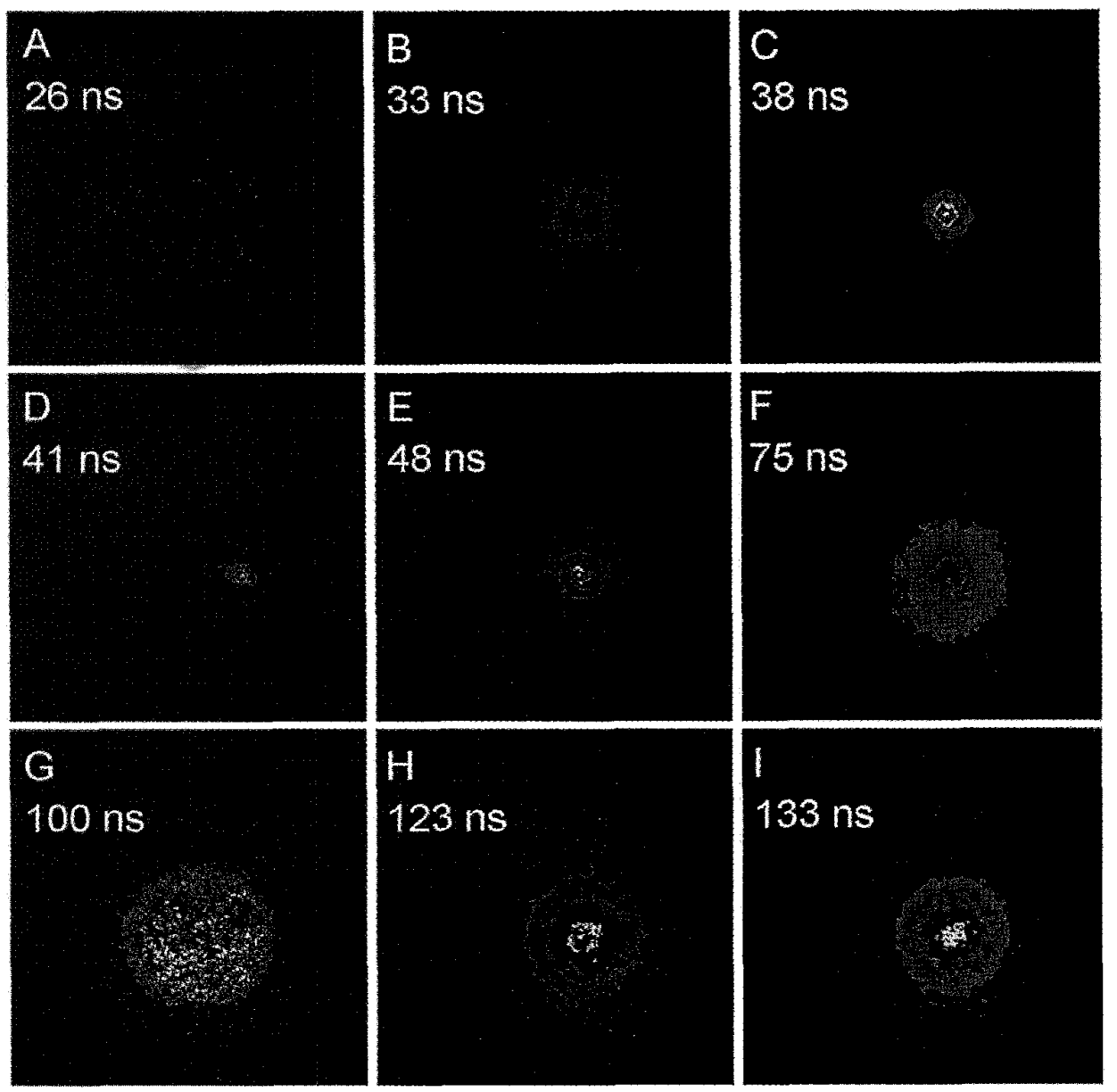

Fig. 1. Sequence of time-resolved soft X-ray pinhole images of the argon capillary discharge viewed end-on. The timing with respect to the origin of the current pulse is indicated. The capillary diameter was $4 \mathrm{~mm}$ and its length was $12 \mathrm{~cm}$. The current pulses had an amplitude of approximately $39 \mathrm{kA}$ and a first half period of $62 \mathrm{~ns}$. The argon pressure was 700 mtorr. The images corresponding to times near and shortly after the time of maximum compression [(D), (E)] were acquired with reduced sensitivity to avoid saturation of the detector.

compressional discharges, and the highest length to diameter ratio $(d / l=500-1000)$ among soft X-ray lasers. These results open an avenue toward the development of a new class of more compact, simpler, and potentially efficient soft X-ray lasers.

\section{REFERENCES}

[1] D. L. Matthews, P. L. Hagelstein, M. D. Rosen, M. J. Eckart, N. M Ceglio, A. N. Hazi, M. Medecki, B. J. MacGowan, J. E. Trebes, B. L. Whitten, E. M. Campbell, C. W. Hatcher, A. M. Hawryluk, R. L. Kaufman, L. P. Pleasance, G. Rambach, J. H. Scofield, G. Stone, and T. A. Weaver, "Demonstration of a soft X-ray amplifier," Phys. Rev. Lett., vol. 54 , p. $1101,1985$.
[2] S. Suckewer, C. H. Skinner, H. Milchberg, C. Keane, and D. Voorhees, "Amplification of stimulated soft X-ray emission in a confined plasma column," Phys. Rev. Lett., vol. 55, p. 1753, 1985.

[3] J. J. Rocca, V. N. Shlyaptsev, F. G. Tomasel, O. D. Cortazar, H Hartshorn, and J. L. A. Chilla, "Demonstration of a discharge pumped table-top soft X-ray laser," Phys. Rev. Lett., vol. 73, p. 2192, 1994.

[4] J. J. Rocca, F. G. Tomasel, M. C. Marconi, V. N. Shlyaptsev, J. L. A. Chilla, B. T. Szapiro, and G. Giudice, "Discharge-pumped soft X-ray laser in neon-like argon," Phys. Plasmas, vol. 2, p. 2547, 1995.

[5] J. J. Rocca, M. C. Marconi, F. G. Tomasel, V. N. Shlyaptsev, J. L. A. Chilla, and D. P. Clark, "Toward saturation of a discharge pumped soft X-ray amplifier," IEEE J. Select. Topics Quantum Electron., vol. 1, p. 945, 1995.

[6] V. N. Shlyaptsev, J. J. Rocca, and A. L. Osterheld, "Dynamics of a capillary discharge X-ray laser," SPIE J., vol. 2520, p. 365, 1995. 